\title{
Phase-difference haploscope using only one shutter disk
}

\author{
MARTIN J. STEINBACH \\ Atkinson College, York University, Downsview, Ontario, Canada MSJ $2 R 7$
}

\begin{abstract}
A simplified version of a phase-difference haploscope to dissociate visual input to the two eyes is described. The unit requires only one motor and shutter disk. This reduces synchronization difficulties found in versions requiring more than one motor.
\end{abstract}

In studying binocular vision it is often necessary to dissociate the input to the two eyes. Common strategies make use of mirror or prism stereoscopes (and their clinical modification into amblyoscopes and synoptophores) and cross-Polaroid or anaglyph techniques. Stereoscopes generally allow only a narrow field of view. Cross-Polaroid techniques, while not limited in field size, require projection surfaces that preserve the plane of polarization and are severely limited in intensity range. Depending on the type of Polaroid used, as little as $1-\log$ unit of attenuation of light intensity between the eyes can exist. To overcome these shortcomings, some researchers use a phase-difference haploscope. This device consists of four shutters (sectored disks),

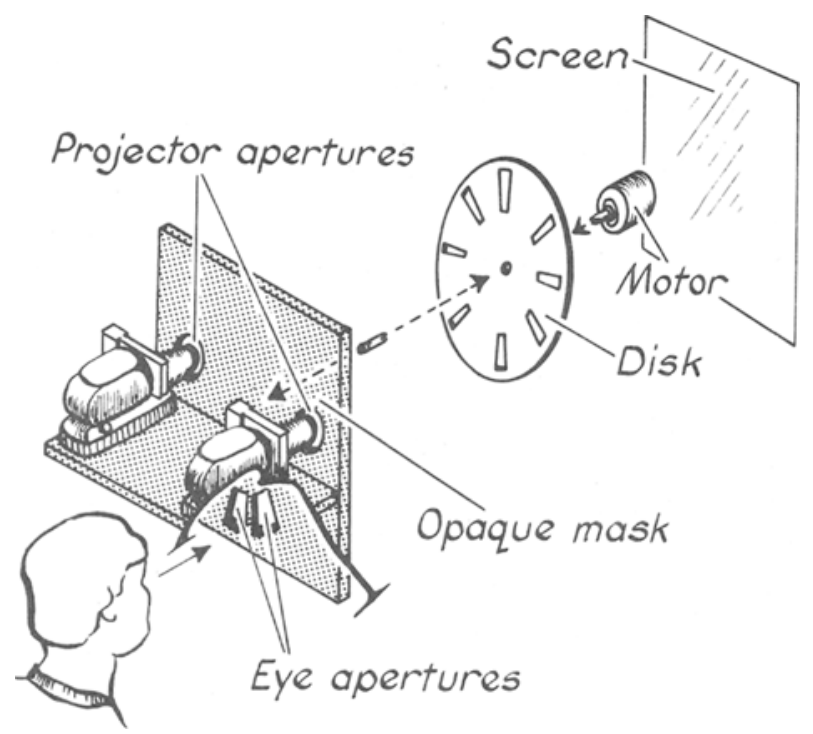

Figure 1. Exploded view of the phase-difference haploscope that uses only one shutter disk. The subject sits close to the mask, eyes aligned with the appropriate eye apertures, and with the motor-driven disk immediately adjacent to the mask.

This device was built at the Smith-Kettlewell Institute of Visual Science, where the author was a Fellow in Strabismus Research. The support of the Smith-Kettlewell Eye Research Foundation and National Research Council of Canada Grant A7664 is gratefully acknowledged. one spinning in front of each eye of the observer and one in front of each of two projectors (e.g., Aulhorn, 1966; Von Noorden, 1970). The phase-difference haploscope does not suffer intensity limitations, and, if the shutter opening is sufficiently large, it is possible to have large field presentations. With the phase properly controlled, light from the left projector enters the left eye only while the right eye and projector are occluded. The right eye and right projector are then simultaneously exposed while the left pair are occluded. The phase-difference haploscope, while simple in concept, requires careful synchronization between the four motors driving the four disks.

The simplified shutter mechanism described here requires only one motor, disk, and mask. With the observer and projectors suitably positioned, the visual inputs to the two eyes can be totally dissociated.

The technique by which the dissociation of input

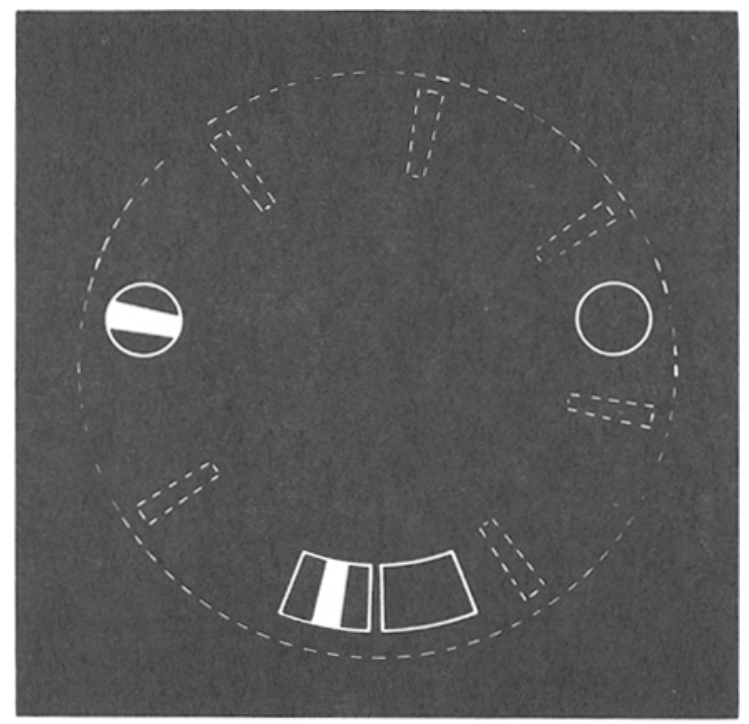

Figure 2. Opaque mask with aperture cut for projectors (circles above) and eyes (rectangles below). The sectored disk (shown in dashed lines) rotates behind the mask and occludes or opens right or left pairs of apertures in turn. In the figure, the left-eye aperture and projector aperture are open: the right pair remain closed. 


\section{ALL MEASUREMENTS IN MILLIMETERS}
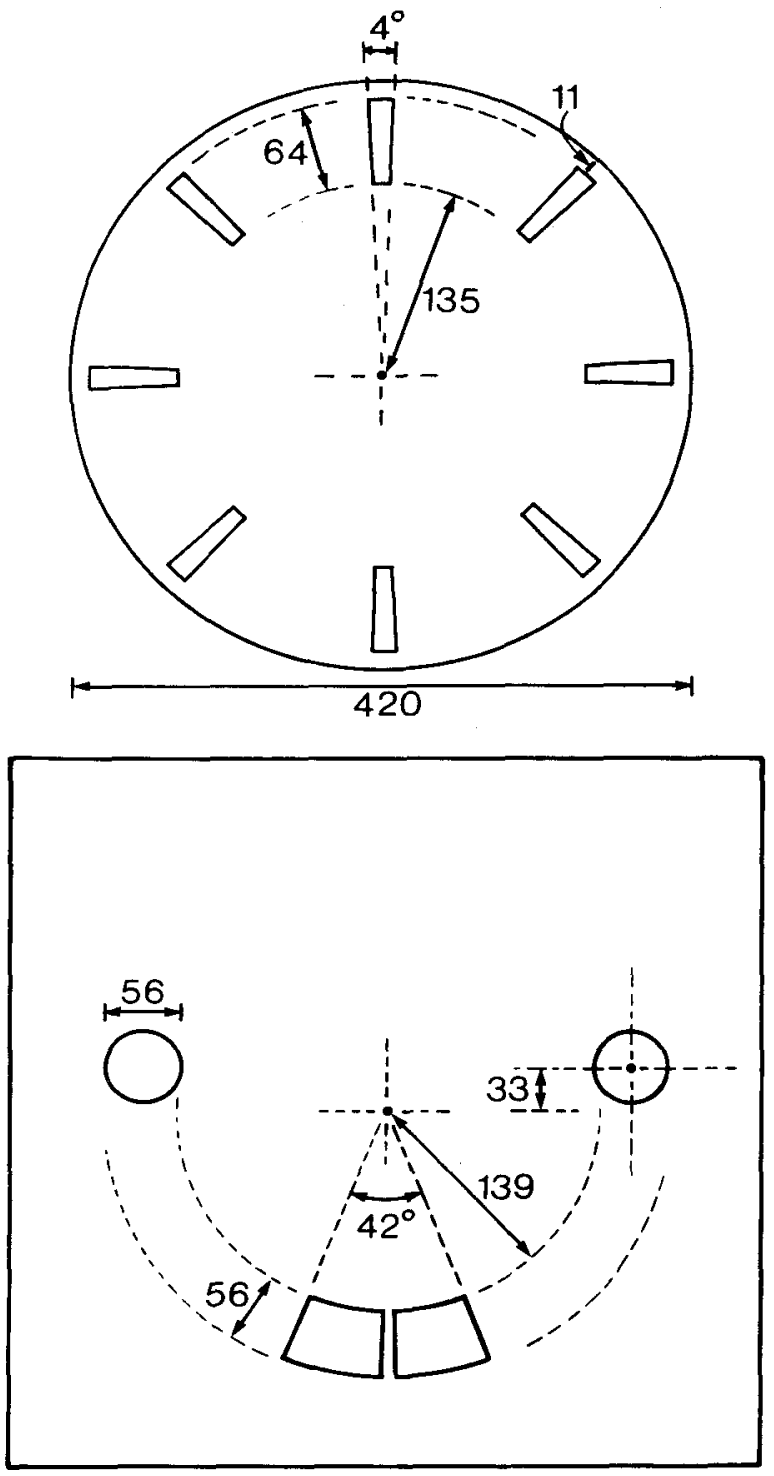

Figure 3. Dimensions for mask and disk. Any thin, opaque, and reasonably rigid material can be used.

between the two eyes is achieved is illustrated in Figure 1, which shows the arrangement of the projectors, subject, shutter disk, and mask. Figure 2 is a schematic description of the shutter disk and opaque mask. The solid white lines represent holes cut in the mask. The dotted lines represent the disk located behind the mask, from the subject's point of view. The upper circular apertures are for the projectors and the two rectangular cutouts at the bottom are for the observers' eyes. Note that the left-eye and left-projector apertures open simultaneously, while the right-eye and rightprojector apertures remain closed. As the disk continues to rotate, the right pair of apertures open with the simultaneous closing of the left pair.

Dimensions for the disk and mask are provided in Figure 3. Different versions were constructed of heavy cardboard (Bristol board) or thin sheet plastic. But any lightweight, opaque, reasonably rigid material will do. The disk was driven by a variable-speed dc motor. For the presentation rate to be beyond flicker fusion, an eight-sectored disk need rotate at 7 or $8 \mathrm{rps}$.

Stereoscopic pairs of stimuli were projected by this method and binocularly normal subjects found it easy to fuse and obtain stereopsis. Random dot stereograms (Julesz, 1971) were readily perceived in three dimensions. Using strabismics, suppression scotomas (Jampolsky, 1955) were mapped, although careful alignment of the eyes and projectors was critical.

In the version of the haploscope described above, no allowance is made for adjustment of interpupillary distance. This distance can be adjusted by altering aperture positions for the projectors so that they open earlier or later with respect to eye opening. This modification can be built in if large interpupillary distance variations in subject population are anticipated.

\section{REFERENCES}

Aulhorn, E. Phasendifferenz-Haploskopie. Klinische Monatsblätter für Augenheilkunde, 1966, 148, 540-544.

JAMPOLSKY, A. Characteristics of suppression in strabismus. Archives of Ophthalmology, 1955, 54, 683-6.

Julesz, B. Foundations of cyclopean perception. Chicago: University of Chicago Press, 1971.

VoN Noorden, G. K. Etiology and pathogenesis of fixation anomalies in strabismus. I: Relationship between eccentric fixation and anomalous retinal correspondence. American Journal of Ophthalmology, 1970, 69, 210-222.

(Received for publication December 10, 1976; revision accepted March $15,1977$. ) 